\title{
Recent progress on the chiral unitary approach to meson meson and meson baryon interactions
}

E. Oset ${ }^{1,2}$, J. A. Oller ${ }^{2}$, J. R. Peláez ${ }^{3}$, A. Ramos ${ }^{4}$, H. C. Chiang $^{5}$, F. Guerrero ${ }^{2}$, S. Hirenzaki ${ }^{6}$, T. S. H. Lee ${ }^{7}$, E. Marco ${ }^{1,2}$, J. C. Nacher ${ }^{1,2}$, Y. Okumura ${ }^{6}$, A. Parreño ${ }^{8}$, H. Toki ${ }^{1}$, M. VicenteVacas $^{2}$

${ }^{1}$ Research Center for Nuclear Physics (RCNP), Osaka University, Ibaraki, Osaka 567-0047, Japan.

${ }^{2}$ Departamento de Física Teórica and IFIC, Centro Mixto Universidad de Valencia-CSIC 46100 Burjassot (Valencia), Spain.

${ }^{3}$ Departamento de Física Teórica, Universidad Complutense, Madrid, Spain.

${ }^{4}$ Departament d'Estructura $i$ Constituents de la Materia, Universitat de Barcelona, Diagonal 647, 08028 Barcelona, Spain.

${ }^{5}$ Institute of High Energy Physics, Academia Sinica, Beijing, China.

${ }^{6}$ Physics Department, Nara Women's University, Japan.

7 Argonne National Laboratory, Argonne, U.S.A.

${ }^{8}$ Institute of Nuclear Theory, University of Washington, Seattle, U.S.A.

\begin{abstract}
We report on recent progress on the chiral unitary approach, analogous to the effective range expansion in Quantum Mechanics, which is shown to have a much larger convergence radius than ordinary chiral perturbation theory, allowing one to reproduce data for meson meson interaction up to $1.2 \mathrm{GeV}$. Applications to physical processes so far unsuited for a standard chiral perturbative approach are presented. Results for the extension of these ideas to the meson baryon sector are discussed, together with applications to kaons in a nuclear medium and $K^{-}$atoms.
\end{abstract}

\section{Invited Talk Presented at the KEK Tanashi Symposium on \\ Physics of Hadrons and Nuclei \\ Tokyo, December 1998 \\ In honor of Prof. K. Yazaki.}




\section{Chiral Unitary Approach}

Chiral perturbation theory $(\chi P T)$ has proved to be a very suitable instrument to implement the basic dynamics and symmetries of the meson meson and meson baryon interaction [1] at low energies. The essence of the perturbative technique, however, precludes the possibility of tackling problems where resonances appear, hence limiting tremendously the realm of applicability. The method that we expose naturally leads to low lying resonances and allows one to face many problems so far intractable within $\chi P T$.

The method incorporates new elements: 1) Unitarity is implemented exactly; 2) It can deal with coupled channels allowed with pairs of particles from the octets of stable pseudoscalar mesons and $\left(\frac{1}{2}^{+}\right)$baryons; 3) A chiral expansion in powers of the external four-momentum of the lightests pseudoscalars is done for $\operatorname{Re} T^{-1}$, instead of the $T$ matrix itself which is the case in standard $\chi P T$.

We sketch here the steps involved in this expansion for the meson meson interaction. One starts from a $K$ matrix approach in coupled channels where unitarity is automatically fulfilled and writes

$$
T^{-1}=K^{-1}-i \sigma,
$$

where $T$ is the scattering matrix, $K$ a real matrix in the physical region and $\sigma$ is a diagonal matrix which measures the phase-space available for the intermediate states

$$
\sigma_{n n}(s)=-\frac{k_{n}}{8 \pi \sqrt{s}} \theta\left(s-\left(m_{1 n}+m_{2 n}\right)^{2}\right),
$$

where $k_{n}$ is the on shell CM momentum of the meson in the intermediate state $n$ and $m_{1 n}, m_{2 n}$ are the masses of the two mesons in the state $n$. The meson meson states considered here are $K \bar{K}, \pi \pi, \pi \eta, \eta \eta, \pi K, \pi \bar{K}, \eta K, \eta \bar{K}$. Since $K$ is real, from eq. (1) one sees that $K^{-1}=\operatorname{Re} T^{-1}$. In non-relativistic Quantum Mechanics, in the scattering of a particle from a potential, it is possible to expand $K^{-1}$ in powers of the momentum of the particle at low energies as follows (in the s-wave for simplicity)

$$
\operatorname{Re} T^{-1} \equiv K^{-1}=\sigma \cdot \operatorname{ctg} \delta \propto-\frac{1}{a}+\frac{1}{2} r_{0} k^{2},
$$

with $k$ the particle momentum, $a$ the scattering length and $r_{0}$ the effective range.

The ordinary $\chi \mathrm{PT}$ expansion up to $O\left(p^{4}\right)$ is given by [1]

$$
T=T_{2}+T_{4}
$$

where $T_{2}$, which is $O\left(p^{2}\right)$, is obtained from the lowest order chiral Lagrangian, $L^{(2)}$, whereas $T_{4}$ contains one loop diagrams in the s, t, u channels, constructed from the lowest order Lagrangian, tadpoles and the finite contribution from 
the tree level diagrams of the $L^{(4)}$ Lagrangian. This last contribution, after a suitable renormalization, is just a polynomial, $T^{(p)}$. Our $T$ matrix, starting from eq. (1) is given by

$$
T=\left[\operatorname{Re} T^{-1}-i \sigma\right]^{-1} \equiv T_{2}\left[T_{2} \operatorname{Re} T^{-1} T_{2}-i T_{2} \sigma T_{2}\right]^{-1} T_{2},
$$

where, in the last step, we have multiplied by $T_{2} T_{2}^{-1}$ on the left and $T_{2}^{-1} T_{2}$ on the right for technical reasons. But using standard $\chi \mathrm{PT}$ we obtain the following expansion up to order $O\left(p^{4}\right)$,

$$
T_{2} \operatorname{Re} T^{-1} T_{2}=T_{2}-\operatorname{Re} T_{4} \cdots
$$

and hence, recalling that $\operatorname{Im} T_{4}=T_{2} \sigma T_{2}$, one obtains

$$
T=T_{2}\left[T_{2}-T_{4}\right]^{-1} T_{2},
$$

which is the coupled channel generalization of the inverse amplitude method of $[2]$.

Once this point is reached one has several options to proceed:

a) A full calculation of $T_{4}$ within the same renormalization scheme as in $\chi P T$ can be done. The eight $L_{i}$ coefficients from $L^{(4)}$ are then fitted to the existing meson meson data on phase shifts and inelasticities up to $1.2 \mathrm{GeV}$, where 4 meson states are still unimportant. This procedure has been carried out in $[2,3]$. The resulting $L_{i}$ parameters are compatible with those used in $\chi P T$. At low energies the $O\left(p^{4}\right)$ expansion for $T$ of eq. (7) is identical to that in $\chi P T$. However, at higher energies the nonperturbative structure of eq. (7), which implements unitarity exactly, allows one to extend the information contained in the chiral Lagrangians to much higher energy than in ordinary $\chi$ $\mathrm{PT}$, which is up to about $\sqrt{s} \simeq 400 \mathrm{MeV}$. Indeed it reproduces the resonances present in the $\mathrm{L}=0,1$ partial waves.

b) A technically simpler and equally successful additional approximation is generated by ignoring the crossed channel loops and tadpoles and reabsorbing them in the $L_{i}$ coefficients given the weak structure of these terms in the physical region. The fit to the data with the new $\hat{L}_{i}$ coefficients reproduces the whole meson meson sector, with the position, widths and partial decay widths of the $f_{0}(980), a_{0}(980), \kappa(900), \rho(770), K^{*}(900)$ resonances in good agreement with experiment [4]. A cut off regularization is used in [4] for the loops in the s-channel. By taking the loop function with two intermediate mesons

$$
G_{n n}(s)=i \int \frac{d^{4} q}{(2 \pi)^{4}} \frac{1}{q^{2}-m_{1 n}^{2}+i \epsilon} \frac{1}{(P-q)^{2}-m_{2 n}^{2}+i \epsilon},
$$

where $P$ is the total meson meson momentum, one immediately notices that

$$
\operatorname{Im} G_{n n}(s)=\sigma_{n n} .
$$

Hence, we can write

$$
\operatorname{Re} T_{4}=T_{2} \operatorname{Re} G T_{2}+T_{4}^{(p)},
$$


where $\operatorname{Re} G$ depends on the cut off chosen for $|\vec{q}|$. This means that the $\hat{L}_{i}$ coefficients of $T_{4}^{(p)}$ depend on the cut off choice, much as the $L_{i}$ coefficients in $\chi P T$ depend upon the regularization scale.

c) For the $\mathrm{L}=0$ sector (also in $\mathrm{L}=0, \mathrm{~S}=-1$ in the meson baryon interaction) a further technical simplification is possible. In these cases it is possible to choose the cut off such that, given the relation between $\operatorname{Re} G$ and $T_{4}^{(p)}$, this latter term is very well approximated by $\operatorname{Re} T_{4}=T_{2} \operatorname{Re} G T_{2}$. This is impossible in those cases because of the predominant role played by the unitarization of the lowest order $\chi P T$ amplitude, which by itself leads to the low lying resonances, and because other genuine QCD resonances appear at higher energies.

In such a case eq. (5) becomes

$$
T=T_{2}\left[T_{2}-T_{2} G T_{2}\right]^{-1} T_{2}=\left[1-T_{2} G\right]^{-1} T_{2},
$$

or, equivalently,

$$
T=T_{2}+T_{2} G T,
$$

which is a Bethe-Salpeter equation with $T_{2}$ and $T$ factorized on shell outside the loop integral, with $T_{2}$ playing the role of the potential. This option has proved to be successful in the $\mathrm{L}=0$ meson meson sector in [5] and in the $\mathrm{L}=$ $0, \mathrm{~S}=-1$ meson baryon sector in $[6]$.

In the meson baryon sector with $\mathrm{S}=0$, given the disparity of the masses in the coupled channels $\pi N, \eta N, K \Sigma, K \Lambda$, the simple "one cut off approach" is not possible. In [7] higher order Lagrangians are introduced while in [8] different subtraction constants (or equivalently different cut offs) in $\mathrm{G}$ are incorporated in each of the former channels leading in both cases to acceptable solutions when compared with the data.

\section{Applications for processes involving pairs of mesons.}

Given the shortness of space we shall not show results on the meson meson and meson baryon scattering that can be found in $[4,6]$, together with all other technical details. Instead, we make a short summary of applications of these ideas to other processes with results for the latest developments.

One of the applications in the meson meson sector is the study of the $\gamma \gamma \rightarrow \pi^{+} \pi^{-}, \pi^{0} \pi^{0}, K^{+} K^{-}, K^{0} \bar{K}^{0}, \pi \eta$ reactions. The $\gamma \gamma \rightarrow \pi^{+} \pi^{-}, \pi^{0} \pi^{0}$ reaction has been one of the standard places to test $\chi P T[9]$, with the obvious limitations to small energies. The new techniques have allowed to extend the calculations up to about $\sqrt{s}=1.4 \mathrm{GeV}$ and include the $K^{+} K^{-}, K^{0} \bar{K}^{0}, \pi \eta$ channels that were not accessible with $\chi P T$. Results for all these channels are presented in [10] where a good agreement with experiment is found in all cases. 
The decay channels of the $\phi(1020)$ resonance also offer a good testing ground for the chiral unitarity theory. In [11] the $\phi \rightarrow \gamma K^{0} \bar{K}^{0}$ decay channel was studied providing a calculation of the background in future experiments testing $\mathrm{CP}$ violation at $D A \Phi N E$. In a recent work these ideas have been extended to study the $\rho^{0} \rightarrow \pi^{+} \pi^{-} \gamma, \pi^{0} \pi^{0} \gamma$ and $\phi \rightarrow \pi^{+} \pi^{-} \gamma, \pi^{0} \pi^{0} \gamma$ process [12]. The latter proceeds via $K^{+} K^{-}$loops as shown in fig. 1 .

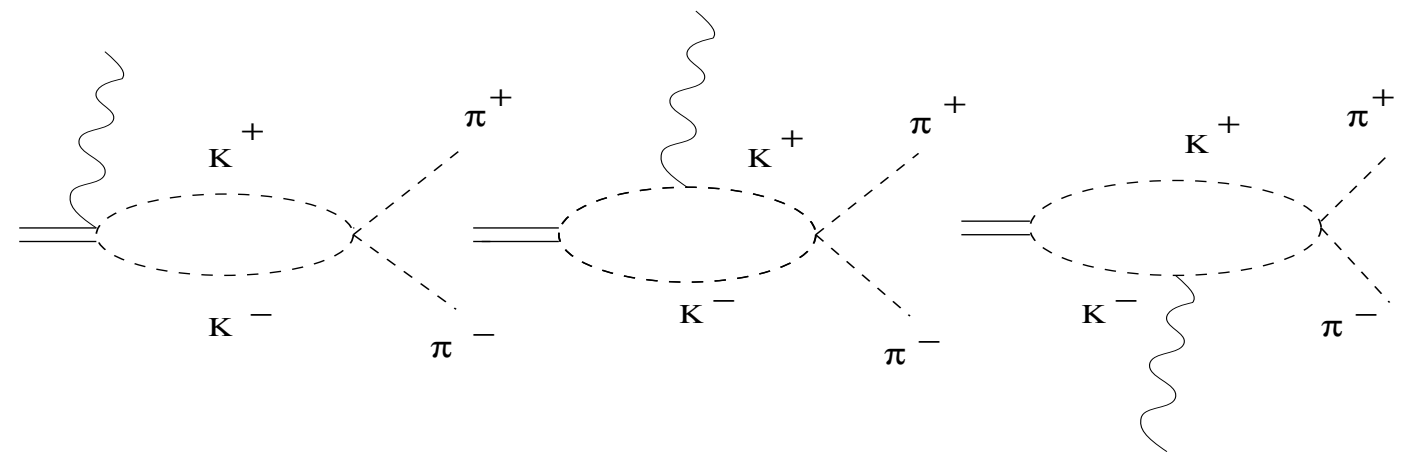

Figure 1: Diagrams contributing to $\phi \rightarrow \pi^{+} \pi^{-} \gamma$ decay.

The transition amplitude for this process behaves as

$$
t^{\gamma} \propto \operatorname{eg} g_{\phi} \tilde{G}\left(M_{\Phi}, M_{I}\right) t_{K^{+} K^{-} \rightarrow \pi^{+} \pi^{-}}\left(M_{I}\right)
$$

where $g_{\phi}$ is the $\phi \rightarrow K^{+} K^{-}$decay coupling, $M_{I}$ the invariant mass of the $\pi^{+} \pi^{-}$system and $\tilde{G}$ sums the loop functions of the three diagrams, which using arguments of gauge invariance one can prove to be finite. At the same time $t_{K^{+} K^{-} \rightarrow \pi^{+} \pi^{-}}\left(M_{I}\right)$, as shown in [11], is the on shell strong scattering matrix for $K^{+} K^{-} \rightarrow \pi^{+} \pi^{-}$, which is evaluated using the chiral unitary techniques in $[12]$.

The branching ratio $\Gamma_{\pi^{+} \pi^{-} \gamma} / \Gamma_{\phi}$ obtained is $2.610^{-4}$ while the $f_{0}$ peak contributes $0.7610^{-4}$. This latter value is compatible and close to the latest boundaries of $B<1-7 \cdot 10^{-4}$ obtained in Novosibirsk [13]. After completion of this work a publication has appeared [14, where the spectrum for $\phi \rightarrow \pi^{0} \pi^{0} \gamma$ is measured. Taking into account that the rate for this process is one half of the one for $\phi \rightarrow \pi^{+} \pi^{-} \gamma$, the value for the branching ratio which are obtain is $1.310^{-4}$ which compares very well with the experimental value $(1.14 \pm$ $0.10 \pm 0.12) 10^{-4}$. The invariant mass distribution is also in agreement with the experimental one, within statistical and systematic errors, and this latter distribution shows clearly the $f_{0}$ peak as predicted by theory. In fig. 2 we show our predicted results compared with the recent measurements of [14]. The agreement found with the whole spectrum is a good support for the chiral unitary theory used. 


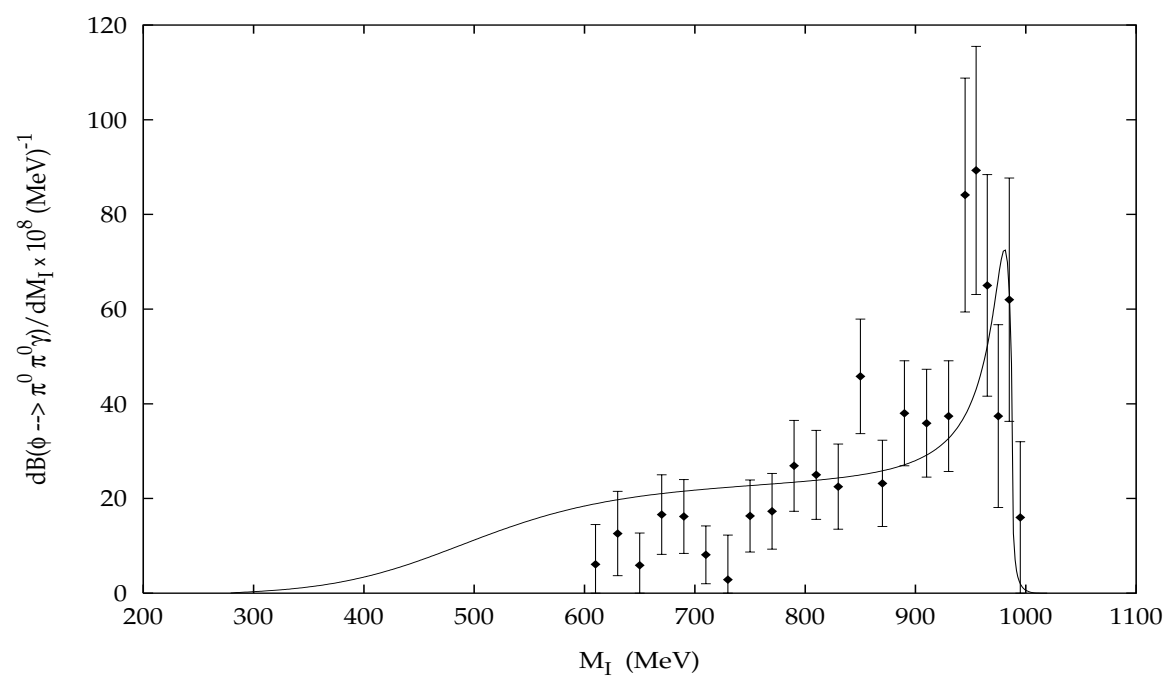

Figure 2: $d B / d M_{I}$ for $\phi \rightarrow \pi^{0} \pi^{0} \gamma$, with $M_{I}$ the invariant mass of the $\pi^{0} \pi^{0}$ system. Experimental points from [14]. Only statistical errors are shown.

Another recent calculation is the reaction $\gamma p \rightarrow f_{0}\left(a_{0}\right) p$ close to threshold [15], which requires energies of $E_{\gamma} \simeq 1.7 \mathrm{GeV}$, or larger, and which can be studied in planned experiments at SPring8/RCNP [16] or TJNAF.

The scattering of mesons in a nuclear medium is an interesting problem and predictions of a quasibound $\pi \pi$ system just below threshold have been made [17]. Interestingly, an enhancement in the mass distribution of two pions close to threshold in the $\pi^{-} A \rightarrow \pi^{+} \pi^{-} A^{\prime}$ reaction is seen [18] which is absent in the $\pi^{+} A \rightarrow \pi^{+} \pi^{+} A^{\prime}$. The calculations done in [19] using the chiral unitary model and medium modifications lead to an appreciable enhancement of the strength around the two pion threshold, although peaks corresponding to the bound state do not appear. The results are remarkably similar to those obtained using a meson exchange picture for the $\pi \pi$ interaction, yet imposing minimal chiral constraints [20], hence stressing the role of chiral symmetry in this process.

Another interesting development along these lines is the work of [21], where, by taking the lowest order chiral Lagrangian and assuming that higher orders of the chiral Lagrangian are generated by the exchange of genuine resonances which survive in the large $N_{c}$ limit [22], the unitary approach together with the comparison with data, allows one to distinguish between the genuine resonances corresponding to QCD states, surviving in the large $N_{c}$ limit, and scattering resonances which appear only as a consequence of the nature of the lowest order Lagrangian together with unitarity.

These are only examples of problems which can be tackled with the new techniques. Given the broad range of applications of $\chi P T$ and the increased range of energies now accessible with the chiral unitary approach, one can envisage a fruitful field of applications with the new approach in strong, weak and electromagnetic processes at intermediate energies. 


\section{Applications in the meson baryon sector}

As quoted above, a good description of the $K^{-} p$ and coupled channel interaction is obtained in terms of the lowest order Lagrangians and the Bethe Salpeter equation with a single cut off. One of the interesting features of the approach is the dynamical generation of the $\Lambda(1405)$ resonance just below the $K^{-} p$ threshold. The threshold behavior of the $K^{-} p$ amplitude is thus very much tied to the properties of this resonance. Modifications of these properties in a nuclear medium can substantially alter the $K^{-} p$ and $K^{-}$nucleus interaction and experiments looking for these properties are most welcome. Some electromagnetic reactions appear well suited for these studies. Application of the chiral unitary approach to the $K^{-} p \rightarrow \gamma \Lambda, \gamma \Sigma^{0}$ reactions at threshold has been carried out in [23] and a fair agreement with experiment is found. In particular one sees there that the coupled channels are essential to get a good description of the data, increasing the $K^{-} p \rightarrow \gamma \Sigma^{0}$ rate by about a factor 16 with respect to the Born approximation.

In a recent paper [24] we propose the $\gamma p \rightarrow K^{+} \Lambda(1405)$ reaction as a means to study the properties of the resonance, together with the $\gamma A \rightarrow$ $K^{+} \Lambda(1405) A^{\prime}$ reaction to see the modification of its properties in nuclei. The resonance $\Lambda(1405)$ is seen in its decay products in the $\pi \Sigma$ channel, but as shown in [24] the sum of the cross sections for $\pi^{0} \Sigma^{0}, \pi^{+} \Sigma^{-}, \pi^{-} \Sigma^{+}$production has the shape of the resonance $\Lambda(1405)$ in the $I=0$ channel. Hence, the detection of the $K^{+}$in the elementary reaction, looking at $d \sigma / d M_{I}\left(M_{I}\right.$ the invariant mass of the meson baryon system which can be induced from the $K^{+}$momentum), is sufficient to get a clear $\Lambda(1045)$ signal. In nuclear targets Fermi motion blurs this simple procedure (just detecting the $K^{+}$), but the resonance properties can be reconstructed by observing the decay products in the $\pi \Sigma$ channel. In fig. 3 we show the cross sections predicted for the $\gamma p \rightarrow K^{+} \Lambda(1405)$ reaction looking at $K^{+} \pi^{0} \Sigma^{0}, K^{+}$all and $K^{+} \Lambda(1405)$ (alone). All of them have approximately the same shape and strength given the fact that the $\mathrm{I}=1$ contribution is rather small.

The energy chosen for the photon is $E_{\gamma}=1.7 \mathrm{GeV}$ which makes it suitable of experimentation at SPring8/RCNP, where the experiment is planned [15], and TJNAF.

One variant of this reaction is its time reversal $K^{-} p \rightarrow \Lambda(1405) \gamma$. This reaction, for a $K^{-}$momentum in the 300 to $500 \mathrm{MeV} / \mathrm{c}$ range, shows clearly the $\Lambda(1405)$ resonant production [25] and has the advantage that the analogous reaction in nuclei still allows the observation of the $\Lambda(1405)$ resonance with the mere detection of the photon, the Fermi motion effects being far more moderate than in the case of the $\gamma A \rightarrow K^{+} \Lambda(1405) X$ reaction which requires larger photon momenta and induces a broad distribution of $M_{I}$ for a given $K^{+}$ momentum.

One of the interesting developments around these lines is the interaction of 


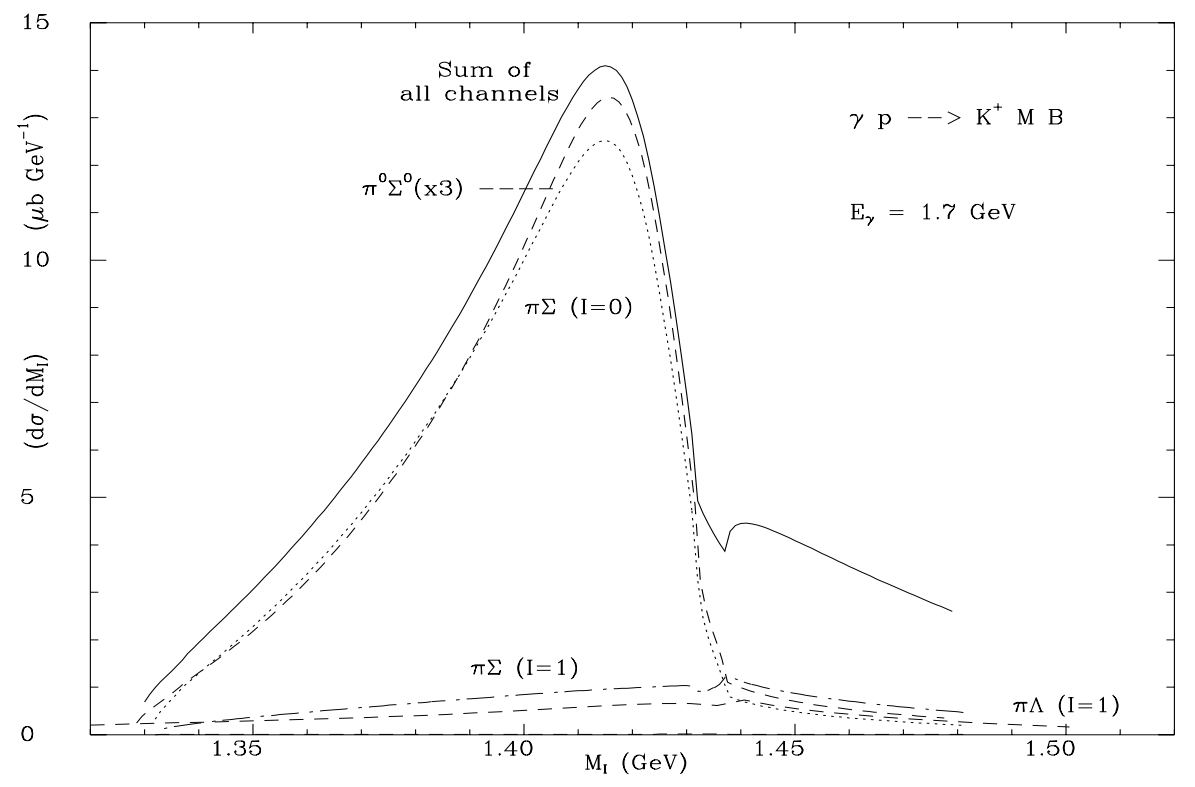

Figure 3: Cross section for $\gamma p \rightarrow K^{+} X$ with $X=$ all, $\pi^{0} \Sigma^{0}, \Lambda(1405)$.

the $K^{-}$with the nuclei, with its relationship to problems like $K^{-}$atoms or the possible condensation of $K^{-}$in neutron stars. The problem has been looked at from the chiral perspective by evaluating Pauli blocking effects on the nucleons of the intermediate $\bar{K} N$ states [26,27]. These effects lead to a $K^{-}$self-energy in nuclei which is attractive already at very low densities, as a consequence of pushing the resonance at energies above $K^{-} p$ threshold. However, more recent investigations considering the $\bar{K}$ self-energy in a self-consistent way [28] lead to quite different results since the resonance barely changes its position. Yet one still gets an attractive self-energy which is demanded by the $K^{-}$atom data [29]. A step forward in this direction is given in [30], where in addition to the $K^{-}$self-energy in the medium, one also renormalizes the pions and takes into account the different binding of $\mathrm{N}, \Sigma$ and $\Lambda$ in nuclei. Preliminary results from [31] indicate that the $K^{-}$self-energy obtained in [30] can lead to a good microscopical description of present data on $K^{-}$atoms, hence providing an accurate tool to study the properties of $K^{-}$at higher densities and the eventual condensation in neutron stars.

\section{Summary}

We have reported on the unitary approach to meson meson and meson baryon interaction using chiral Lagrangians, which has proved to be an efficient method to extend the information contained in these Lagrangians to higher energies where $\chi P T$ cannot be used. This new approach has opened the doors to the investigation of many problems so far intractable with $\chi P T$ and a few ex- 
amples have been reported here. It is clear that these are only a few of the many and interesting problems which can now be tackled from this perspective. At the same time we have shown that many interesting predictions can be tested with present machines, and a few experiments in this direction are already planned. Further research, theoretical and experimental, along these lines appears to us a very interesting task to undertake in a near future.

\section{Acknowledgments.}

We are thankful to the COE Professorship program of Monbusho which enabled E. O. to stay at RCNP where part of the work reported here has been done. E. M. and J. C. N. would like to acknowledge the hospitality of the RCNP of the Osaka University and support from the Ministerio de Educacion

y Cultura. This work is partly supported by DGICYT, contract number PB 96-0753.

\section{References}

[1] J. Gasser and H. Leutwyler, Ann. Phys. 158(1984)142; Nucl. Phys. B250(1985)465

[2] A. Dobado, M. J. Herrero and T. N. Truong, Phys. Lett. B235(1990)134;

A. Dobado and J. R. Peláez, Phys. Rev. D47(1993)4883

[3] F. Guerrero and J. A. Oller, Nucl. Phys. B 537(1999)459

[4] J. A. Oller, E. Oset and J. R. Peláez, Phys. Rev. Lett. 80(1998)3452; Phys. Rev. D in print hep-ph/9804209

[5] J. A. Oller and E. Oset, Nucl. Phys. A620(1997)438; erratum Nucl. Phys. $\mathrm{A}$ in print

[6] E. Oset and A. Ramos, Nucl. Phys. A635(1998)99

[7] N. Kaiser, T. Waas and W. Weise, Nucl. Phys. A612 (1997) 297

[8] A. Parreño, A. Ramos and E. Oset, in preparation; A. Parreño, poster session in the Conference

[9] J. Bijnens and F. Cornet, Nucl. Phys. B296(1988)557; J. F. Donaghue, B. K. Holstein and Y. C. Lin, Phys. Rev D37(1998)2423

[10] J. A. Oller and E. Oset, Nucl. Phys. A629(1998)739

[11] J. A. Oller, Phys. Lett. B426(1998)7

[12] E. Marco, S. Hirenzaki, E. Oset and H. Toki, RCNP preprint 1999 
[13] R. R. Akhmetshin et al., Phys. Lett. B415(1997)452

[14] M. N. Achasov et al., Phys. Lett. B440(1998)442

[15] E. Marco, E. Oset and H. Toki, RCNP preprint 1998.

[16] T. Nakano, talk in this Conference.

[17] P. Schuk, W. Nörenberg and G. Chanfray, Z. Phys. A330(1988)119

[18] F. Bonutti et al., Phys. Rev. Lett. 77(1996)603

[19] H. C. Chiang, E. Oset and M. J. Vicente Vacas, Nucl. Phys. A644(1998)77

[20] Z. Aouissat et al., nucl-th/9806069

[21] J. A. Oller and E. Oset, submitted to Phys. Rev. D, hep-ph/9809337

[22] G. Ecker, J. Gasser, A. Pich and E. de Rafael, Nucl. Phys. B321(1989)311

[23] T. S. H. Lee, J. A. Oller, E. Oset and A. Ramos, Nucl. Phys A643(1998)402

[24] J. C. Nacher, E. Oset, H. Toki and A. Ramos, RCNP preprint, nuclth/9812055

[25] J. C. Nacher, E. Oset, H. Toki and A. Ramos, RCNP preprint 1999

[26] V. Koch, Phys. Lett. B337(1994)7

[27] T. Waas, N. Kaiser and W. Weise, Phys. Lett. B365(1996)12; B379(1996)34; T. Waas and W. Weise, Nucl. Phys. A625(1997)287

[28] M. Lutz, Phys. Lett. B426(1998)12

[29] C. J. Batty, E. Friedman and A. Gal, Phys. Rep. 287(1997)385

[30] A. Ramos and E. Oset in preparation

[31] Y. Okumura, S. Hirenzaki, H. Toki, A. Ramos and E. Oset in preparation 